\title{
THE INDEPENDENCE OF CERTAIN DISTRIBUTIVE LAWS IN BOOLEAN ALGEBRAS
}

\author{
BY \\ DANA SCOTT
}

Let $\alpha$ be a regular cardinal number. We shall prove the following:

THEOREM. There is a complete Boolean algebra that is $(\beta, \gamma)$-distributive for every $\beta<\alpha$ and every cardinal $\gamma$, but is not $(\alpha, \alpha)$-distributive $\left.{ }^{(}\right)$.

The method of proof is to construct the desired algebra as the algebra of all regular open sets of a suitable topological space. To this end we note first

Lemma 1. There is a 0 -dimensional Hausdorff space $X$ such that

(i) the class of open sets of $X$ is closed under the formation of $\beta$-termed intersections for every $\beta<\alpha$;

(ii) the class of nowhere-dense sets of $X$ is closed under the formation of $\beta$-termed unions for every $\beta<\alpha$;

(iii) there is an $\alpha \times 2$-termed sequence $C$ of nonempty open-closed sets of $X$ such that

(iii $\left.{ }_{1}\right) C_{\xi 0} \cup C_{\xi 1}=x$ for $\xi<\alpha$; and

(iii $\left.{ }_{2}\right) \bigcap_{\xi<\alpha} C_{\xi f(\xi)}$ is nowhere-dense for $f \in 2^{\alpha}$.

Proof. Let the set of points of the space $x$ be the set of all subsets of $\alpha$. (Notice that $\alpha$ is considered as an ordinal number, and that each ordinal is the set of all smaller ordinals. Thus, for example, every ordinal $\beta<\alpha$ is also a point of $x$.) If $x$ and $y$ are two subsets of $\alpha$, denote by $[x, y]$ the interval of all sets $z$ such that $x \subseteq z \subseteq y$. As a basis for the open sets of $x$ take the collection of all intervals $[x, y]$ such that $x \cup(\alpha-y) \subseteq \beta$ for some $\beta<\alpha$. An empty interval is also included in the basis. Suppose that $\beta<\alpha$ and $\left\{\left[x_{\xi}, y_{\xi}\right]: \xi<\beta\right\}$ is a sequence of basic open sets where $x_{\xi} \cup\left(\alpha-y_{\xi}\right) \subseteq \gamma_{\xi}<\alpha$ for $\xi<\beta$. We have

$$
\bigcap_{\xi<\beta}\left[x_{\xi}, y_{\xi}\right]=\left[\bigcup_{\xi<\beta} x_{\xi}, \bigcap_{\xi<\beta} y_{\xi}\right]
$$

and

$$
\bigcup_{\xi<\beta} x_{\xi} \cup\left(\alpha-\bigcap_{\xi<\beta} y_{\xi}\right) \subseteq \bigcup_{\xi<\beta} \gamma_{\xi} .
$$

From the regularity of $\alpha$ it follows that $U_{\xi<\beta} \gamma_{\xi}<\alpha$; thus the intersection of the sequence of basic open sets is again a basic open set. (Notice that if the

Received by the editors April 4, 1956.

(1) For terminology see Smith-Tarski [3]. This theorem has also been proved in a weaker form in Smith [2]. 
sequence is a decreasing sequence of nonempty sets, then the intersection is nonempty.) An open set then is a union of basic open sets. It is obvious that there are no isolated points in the space $X$ and that $X$ is Hausdorff. Also clear is the proof that every basic open set is closed, showing that $X$ is 0 -dimensional. An easy computation using the set-theoretical distributive law and the fact just established about the intersections of basic open sets yields finally a proof of (i).

Let $\theta$ be the collection of all nonempty basic open sets. Let $\beta<\alpha$ and $N$ be a $\beta$-termed sequence of nowhere-dense sets. To show that $N^{*}=U_{\xi<\beta} N_{\xi}$ is nowhere dense, it suffices to show that for every $Y \in \mathcal{O}$ there is a $Z \in \mathcal{O}$ such that $Z \subseteq Y$ and $Z \cap N^{*}=O$. By the axiom of choice let $\mathcal{e}$ be a function that chooses a set from every nonempty family of subsets of our space. Let $Y \in \mathcal{O}$ and define by recursion a $\beta$-termed sequence $G$ such that for $\xi<\beta$

$$
G_{\xi}=\mathfrak{e}\left\{Z: Z \in \mathcal{O} \text { and } Z \subseteq Y \cap \bigcap_{\eta \xi \xi} G_{\eta} \text { and } Z \cap N_{\xi}=0\right\} .
$$

We proceed by induction to show that this sequence is well-defined. Thus suppose that $G_{\eta}$ is well-defined for all $\eta<\xi$ where $\xi<\beta$. It is clear that the sequence is decreasing up to this point and hence $Y \cap \cap_{\eta<\xi} G_{\eta} \in \mathcal{O}$. The fact that $N_{\xi}$ is nowhere-dense implies that there is a $Z \in \mathcal{O}$ such that $Z \subseteq Y \cap \bigcap_{\eta<\xi} G_{\eta}$ and $Z \cap N_{\xi}=O$. It follows at once that $G_{\xi}$ is well-defined. It is obvious now that the whole sequence $G$ is decreasing, and hence $G^{*}=\bigcap_{\xi<\beta} G_{\xi} \in \mathcal{O}$ and $G^{*} \subseteq Y$ and $G^{*} \cap N^{*}=O$. This argument shows that $N^{*}$ is nowhere-dense and establishes property (ii).

To prove (iii) we have only to let

$$
C_{\xi 0}=[\{\xi\}, \alpha]
$$

and

$$
C_{\xi 1}=[O, \alpha-\{\xi\}] \quad \text { for } \xi<\alpha .
$$

Since these sets are basic open sets they are also closed. Formula (iii ${ }_{1}$ ) is obvious and $\left(\mathrm{iii}_{2}\right)$ is a consequence of the simple fact that

$$
\bigcap_{\xi<\alpha} C_{\xi f(\xi)}=\left\{f^{-1}(0)\right\} \quad \text { for } f \in 2^{\alpha} .
$$

This completes the proof of Lemma 1.

If $\alpha=\omega$ our space is nothing more than the Cantor Discontinuum. For larger $\alpha$ the space is compact only in the sense that every open cover can be reduced to one of power less than $\alpha$. The proof of (ii) above could easily be modified to show that no nonempty open set is an $\alpha$-termed union of nowheredense sets-the analogue of the Baire Category Theorem. A rather different construction of the space has been given by Sikorski in [1] (see especially p. 129 where the space is called $D_{\mu}$ where $\alpha=\omega_{\mu}$.) Our construction here 
would seem neater since there is no need of any non-Archimedean metric; however, the particular form of the space $X$ is of no importance for the present purpose.

Let $\Re$ be the algebra of all regular open sets of the space $x$. That $\Re$ is a complete Boolean algebra is well-known $\left({ }^{2}\right)$. The Boolean operations of $\Re$ will be denoted by the usual symbols $+, \cdot, \sum, \Pi$. The unit element of $\Re$ is $x$ itself, while the zero element is just the empty set $O$. The next lemma, which we state without proof, relates the Boolean operations in $\Re$ to the settheoretical operations in $x$. We use the symbols in $X$ and $\mathrm{cl} X$ to denote the interior and closure of the set $X$.

Lemma 2. If $\beta$ is any ordinal and $X$ is a $\beta$-termed sequence of regular open sets (i.e. elements of $\Re$ ), then

(i) $\sum_{\xi<\beta} X_{\xi}=$ in $\mathrm{cl} \mathrm{U}_{\xi<\beta} X_{\xi}$;

(ii) $\prod_{\xi<\beta} X_{\xi}=$ in $\operatorname{cl} \bigcap_{\xi<\beta} X_{\xi}$;

(iii) $\sum_{\xi<\beta} X_{\xi}-U_{\xi<\beta} X_{\xi}$ is nowhere-dense;

(iv) $\bigcap_{\xi<\beta} X_{\xi}-\prod_{\xi<\beta} X_{\xi}$ is nowhere-dense.

Lemma 3. $\Re$ is $(\beta, \gamma)$-distributive for every $\beta<\alpha$ and every $\gamma$.

Proof. Let $\beta<\alpha$ and let $\gamma$ be any ordinal. Given a $\beta \times \gamma$-termed sequence $X$ of regular open sets and an open set $A$ satisfying the formula

$$
\sum_{\eta<\gamma} X_{\xi \eta}=A \neq O \quad \text { for each } \xi<\beta,
$$

then we must show that there is a function $f \in \gamma^{\beta}$ such that ${ }^{(3)}$

$$
\prod_{\xi<\beta} X_{\xi f(\xi)} \neq O \text {. }
$$

Thus, by way of contradiction, assume that for all functions $f \in \gamma^{\beta}$

$$
\prod_{\xi<\beta} X_{\xi f(\xi)}=O .
$$

By virtue of Lemma 2 (iv), formula (3) implies

$$
\bigcap_{\xi<\beta} X_{\xi f(\xi)} \text { is nowhere-dense. }
$$

Since $\beta<\alpha$ and each set $X_{\xi_{\eta}}$ is open, we have by Lemma 1 (i)

$$
\bigcap_{\xi<\beta} X_{\xi f(\xi)} \text { is open. }
$$

Formulas (4) and (5) yield at once

(2) See for example Tarski [4]. A subset of a topological space is called a regular open set if it is equal to the interior of its closure.

(3) For the equivalence of this form of the distributive law to other forms see SmithTarski [3, Theorem 2.2]. 


$$
\bigcap_{\xi<\beta} X_{\xi f(\xi)}=O
$$

Hence we can derive from (6) the formula

$$
\bigcup_{f \in \gamma^{\beta}} \bigcap_{\xi<\beta} X_{\xi f(\xi)}=O \text {. }
$$

In view of the general set-theoretical distributive law, (7) implies

$$
\bigcap_{\xi<\beta} \bigcup_{\eta<\gamma} X_{\xi \eta}=O \text {. }
$$

From formula (8) we derive

$$
A=A-\bigcap_{\xi<\beta} \underset{\eta<\gamma}{\bigcup} X_{\xi \eta}=\bigcup_{\xi<\beta}\left(A-\bigcup_{\eta<\gamma} X_{\xi \eta}\right) .
$$

Now by Lemma 2 (iii) and formula (1) we have for each $\xi<\beta$

$$
A-\bigcup_{\eta<\gamma} X_{\xi \eta} \text { is nowhere-dense. }
$$

By virtue of Lemma 1 (ii), formulas (9) and (10) imply that the set $A$ is nowhere-dense, which contradicts the assumption that $A$ is a nonempty open set. The proof of Lemma 3 is thus complete.

Lemma 4. $\Re$ is not $(\alpha, \alpha)$-distributive.

Proof. Clearly the terms of the sequence $C$ of Lemma 1 (iii) are regular open sets. In terms of the Boolean operations of $\Re$ conditions ( $\mathrm{iii}_{1}$ ) and (iii ${ }_{2}$ ) may be written as
(iii)
$C_{\xi 0}+C_{\xi 1}=x$
for $\xi<\alpha$;
(iii')
$\prod_{\xi<\alpha} C_{\xi f(\xi)}=0$
for $f \in 2^{\alpha}$.

Whence we see that the sequence $C$ itself offers a counterexample to the $(\alpha, 2)$-distributive law.

Our theorem is now a direct consequence of Lemmas 3 and 4.

\section{BIBLIOGRAPHY}

1. R. Sikorski, Remarks on some topological spaces of high power, Fund. Math. vol. 37 (1950) pp. 123-136.

2. E. C. Smith, A distributivity condition for Boolean algebras, Ann. of Math. vol. 64 (1956) pp. 551-561.

3. E. C. Smith and A. Tarski, Higher degrees of distributivity and completeness in Boolean algebras, Trans. Amer. Math. Soc. vol. 84 (1957) pp. 230-257.

4. A. Tarski, Les fondements de la géométrie des corps, Ksiegga pamiątkowa Pierwsego Polskiego Zjazdu Mathematycznego Lwów vol. 9 (1927) pp. 29-33.

PRINCETON University,

Princeton, N. J. 